\title{
Unilateral krukenberg tumor can originate from rectosigmoid adenocarcinoma: a case report with normal pre-operative serum CA-I 25 level
}

\begin{abstract}
Background: Krukenberg tumor is a rare metastatic ovarian cancer, usually solid and mostly detected bilaterally. Common primary sites of the tumor are the gastrointestinal (GI) tract and the breast.

Case Presentation: A 42-year old female patient, who was previously diagnosed with a cyst in the right ovary, reported severe pelvic pain, constipation, and rectal bleeding for the last two months. Pre-operative serum CA-125 level was normal. Ultrasound and Computed Tomography (CT) revealed the presence of a mass of cystic and solid elements in the right ovary. The intraoperative detection of a mass in the rectosigmoid led to right oophorectomy with fallopian tube excision, Hartmann's procedure and appendectomy. Histological examination revealed mucinous cystadenocarcinoma of the ovary, while sites of moderately differentiated adenocarcinoma were detected in the colon specimen.
\end{abstract}

Conclusion: This occurrence of unilateral Krukenberg tumor with synchronous colonic adenocarcinoma is quite rare. The rectosigmoid as a primary site has been scarcely reported in the literature.

Keywords: krukenberg tumor, surgery, colon cancer, ovarian metastasis
Volume 9 Issue 4 - 2018

\begin{abstract}
Michail Kirmanidis,' Christodoulos Keskinis, ${ }^{2}$ loannis Kariotis, ${ }^{3}$ Anastasios Chatziaggelou, ${ }^{4}$ Maria Sachanidou ${ }^{5}$

'Department of General Surgery, General Hospital of Drama, Greece

${ }^{2}$ Health Center of Nevrokopi, Greece

${ }^{3}$ Department of General Surgery, General Hospital of Drama, Greece

${ }^{4}$ Department of Obstetrics and Gynecology, General Hospital of Drama, Greece

5Department of ENT, “Gewrgios Gennimatas” General Hospital of Thessaloniki, Greece
\end{abstract}

\begin{abstract}
Correspondence: VMichail A Kirmanidis, Department of General Surgery, General Hospital of Drama, 66100, Terma Ippokratous, Drama, Greece, Tel +30 6974777655, E-mail:mihaliskirmanidis@yahoo.gr
\end{abstract}

Received: May 10, 2018 | Published: July 16, 2018

\section{Introduction}

Krukenberg Tumor (KT) is an infrequent metastatic tumor of the ovary, accounting for $1 \%$ to $2 \%$ of all ovarian tumors..$^{1-3}$ Being a subject of debate for over a century, the KT is mainly considered as a metastatic neoplasm. ${ }^{1,4}$ Despite the fact that two recent studies report the colorectum as the most frequent primary site, the stomach is generally considered as the most common primary site of the disease $(70 \%){ }^{1,5,6}$ Moreover, KT is mainly attributed to carcinomas of the GI tract (appendix, liver, pancreas, biliary tract and gallbladder) and rarely to that of the breast. ${ }^{1,2,3,7,8}$

We report a rare occurrence of rectosigmoid carcinoma as the primary site of a unilateral KT metastasis (right ovary) with normal levels of CA-125. Specific significance with respect to prognosis and decision-making is underscored. ${ }^{1,5,9,10}$

\section{Case report}

A 42-year-old female patient, with history of hemorrhoidopathy and positive history of hepatitis B, was admitted for elective resection of the right ovary, due to the presence of a cyst. Its presence had been documented seven months ago with abdominal ultrasound. Medical history revealed severe pelvic pain, constipation, flatulence and rectal bleeding, as well as blood in stools approximately for the last two months.

The laboratory examination of the patient was as follows: Hemoglobin $=9 \mathrm{~g} / \mathrm{dl}$, Haematocrit $=29.3 \%$ (mild anemia), White blood cells: $8.87 \mathrm{k} / \mathrm{mm}^{3}, C R P=16.90 \mathrm{mg} / \mathrm{dL}$, while all the cancer markers were normal. $(\mathrm{CA}-125=20,1 \mathrm{U} / \mathrm{mL}, \mathrm{CEA}=2.56 \mathrm{ng} / \mathrm{mL}, \mathrm{AFP}=5.10 \mathrm{ng} /$ $\mathrm{mL}, \mathrm{CA}-19.9=34 \mathrm{U} / \mathrm{mL}, \mathrm{CA}-15.3=9.3 \mathrm{U} / \mathrm{mL}$ ).
A new abdominal ultrasound (Figure 1) confirmed the presence of cyst in the right ovary. Computed Tomography (CT) scans were used for further staging and pre-operative evaluation of the mass.

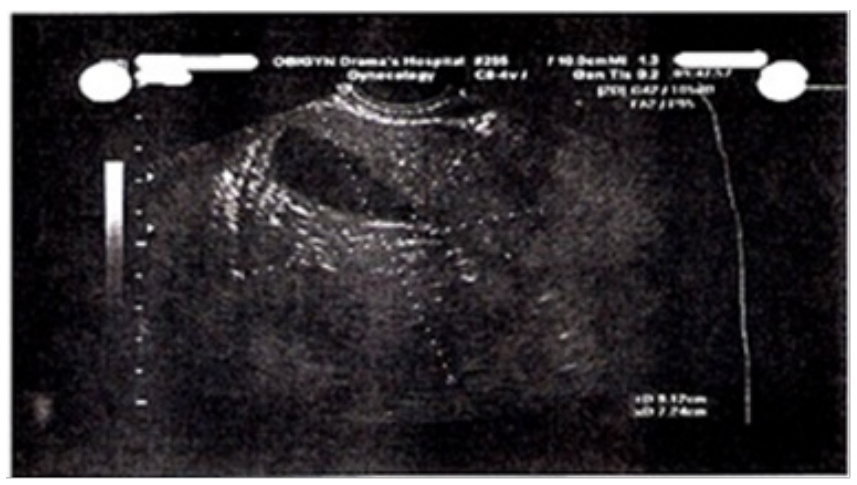

Figure I Abdominal ultrasound shows the cyst presence in the right ovary.

The CT (Figures 2-4) showed a large, multi-spaced, clearly defined circumscribed mass composed of cystic and solid components with a volume of 400 milliliters (maximum diameter: $10.8 \mathrm{~cm}$ ). The mass extended up to the level of the right distal ureter without infiltrating it. Normal lymph nodes (para-aortic space) were noted. No other pathological findings were detected in the abdomen.

The intraoperative findings were in discrepancy with the laboratory tests and the imaging control. In the beginning the patient underwent right oophorectomy and then, a mass was palpated in the rectosigmoid. Hence, the patient underwent an excision of the right fallopian tube as well as resection of the lower sigmoid/ rectosigmoid colon with end colostomy (Hartmann's procedure) and appendectomy. 


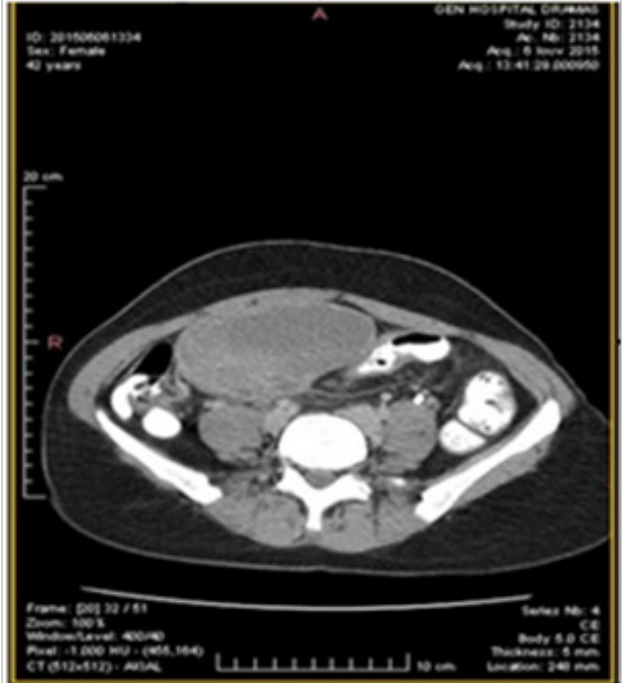

Figure $2 \mathrm{CT}$ abdomen (transverse plane) depicts the cyst presence in the right ovary $(10.5 \mathrm{~cm} \times 7 \mathrm{~cm})$ with solid and cystic elements.

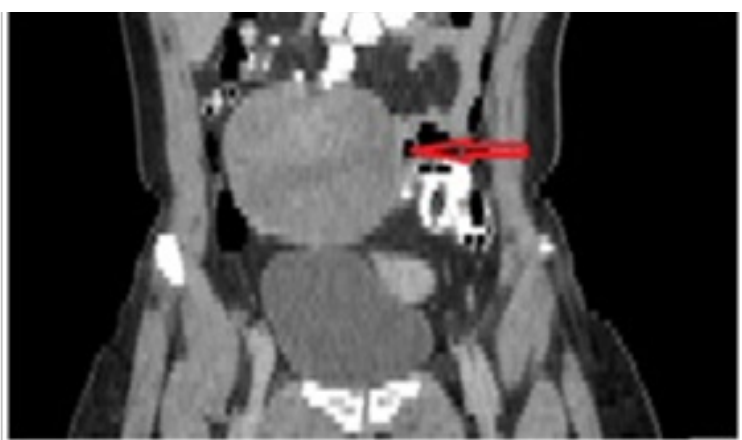

Figure $3 \mathrm{CT}$ abdomen (coronal plane) shows the cyst presence in the right ovary $(10,5 \mathrm{~cm} \times 7 \mathrm{~cm}$ sizes) with solid and cystic elements. The red arrow indicates the lesion.

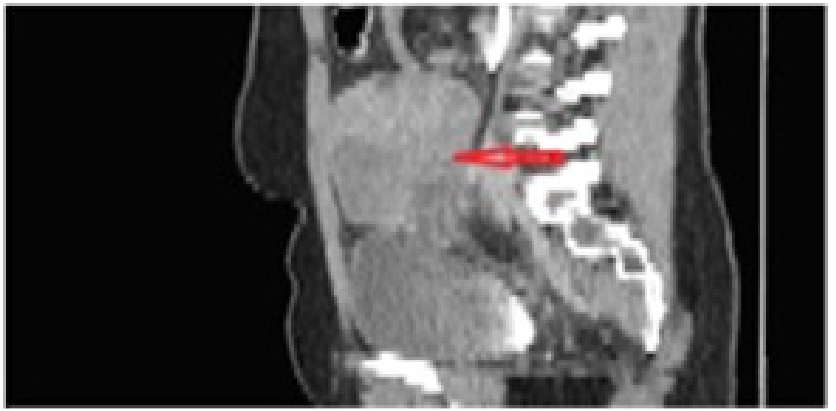

Figure $4 \mathrm{CT}$ abdomen (sagittal plane) shows the cyst presence in the right ovary $(10.5 \mathrm{~cm} \times 7 \mathrm{~cm})$ with solid and cystic elements. The red arrow indicates the lesion.

The specimen of rectosigmoid colon revealed a circumferential tumor, occupying the two-thirds of the intestinal wall and causing partial obstruction of the lumen (Figure 5). In cross section, the tumor appeared to infiltrate the wall and extend to the pericolic adipose tissue. The tumor of the colon was a mucinous adenocarcinoma of good-to-moderate differentiation as well. The tumor cells were immunoreactive for CK20; all of them were negative for CK7, CDX2 and CA 19-9 while a certain percentage was immunoreactive for CA125 staining.

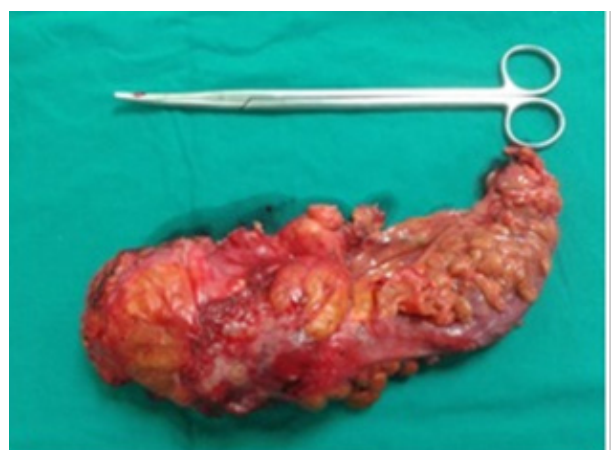

Figure $5 \mathrm{I} 2 \mathrm{~cm}$ surgical specimen of the rectosigmoid colon.

The histology report revealed the right ovary (Figure 6) measuring $0.5 \mathrm{~cm} \times 7 \mathrm{~cm} \times 7.5 \mathrm{~cm}$ in size. The tumor included mucinous adenocarcinoma cancer cells of moderate differentiation without signet ring cells. In addition, the tumor cells were immunoreactive for CK20, focally immunoreactive for CDX2 but negative for CK7, CA-125 and CA-19-9.

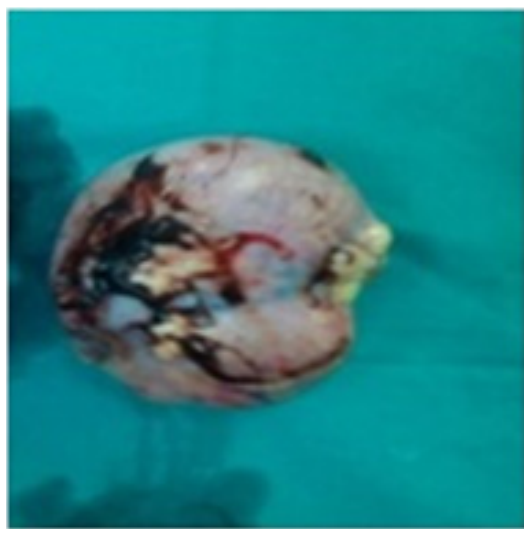

Figure 6 Right ovary measured $10,5 \mathrm{~cm} \times 7 \mathrm{~cm} \times 7,5 \mathrm{~cm}$ in size.

Initially, it was difficult to determine the primary site of the neoplasm, by differential diagnosis, even though the immunohistochemical findings indicated clearly the large intestine as the primary site. ${ }^{3}$

Subsequently, the patient was admitted for adjuvant chemotherapy right after the surgery. She has been undergoing 4 cycles of chemotherapy with capecitabine/irinotecan combination regimen.

\section{Discussion}

In general, more than $80 \%$ of Krukenberg cases appear bilaterally. Bilateral KT usually co-exists with pregnancy and the vast majority is synchronous (70-80\%), ${ }^{2,11-13}$ Additionally, the tumor is usually solid and, even though it is a poorly differentiated intestinal type adenocarcinoma with or without signet ring cells, it often produces mucins. ${ }^{1,2}$

In our case, a synchronous tumor in the rectosigmoid and a unilateral Krukenberg tumor seemed to appear simultaneously. Only two recent case-reports with rectosigmoid as a primary site have been recorded in the literature and were bilateral. ${ }^{9,10}$ This tumor consisted 
of both cystic and solid elements. Moreover, the colon tumor was also a mucinous adenocarcinoma of good- and moderate differentiation without signet ring cells. Our patient was 42 years old, confirming the fact that the disease is usually present within an average range of 40-46 years old. ${ }^{3}$

Half of the patients present ascites as a syptom. ${ }^{1}$ Its absence from our case is theoretically considered to be a good prognostic factor, as its presence usually reveals peritoneal infiltration. ${ }^{1,14}$ However, a recent study indicates that the presence of the ascites has not a remarkable impact on the survival rate for KT with colorectal origin. ${ }^{15}$

The mild anemia and the increased CRP were attributed to the hemorrhoidopathy and to the known presence of the cyst and this is the reason why colonoscopy did not take place preoperatively. The combination of the laboratory and imaging tests did not identify the nature of the cyst in the right ovary. Some newly developed morphological scoring systems are considered helpful in differentiating a benign from a malignant pelvic tumor, playing a crucial role in the prognosis. ${ }^{16}$

The cancer markers were normal (CA-125, CEA, AFP, CA-19.9, CA-15.3), which is very significant given the specific importance and validity of the serum CA-125 level. ${ }^{1,3,17}$ Preoperative serum CA125 levels are pathologically high in most cases before the operation and decrease thereafter. ${ }^{1}$ This marker is used in the postoperative follow-up period to detect an early ovarian metastasis from the gastrointestinal tract. ${ }^{1,3,17}$ TAG-72 can be also useful in the followup control for relapse. ${ }^{18}$ Additionally, a recent study connects the low CA-125 levels $(<75 \mathrm{U} / \mathrm{mL})$ with a favorable 5-year survival rate. ${ }^{1}$ In our patient, serum CA-125 $=20.1 \mathrm{U} / \mathrm{mL}$ level was impressively low, which can be considered as a very good prognosis factor. ${ }^{1,3}$

Our patient was treated with right salpingo-oophorectomy (SO), Hartmann's procedure and appendectomy. In our case, the patient underwent an appendectomy as an extra precautionary treatment and not because it was considered as a possible primary site of the ovarian tumor, even though it sometimes can be. ${ }^{19-21}$ Notably, a universal agreement regarding the proper surgical treatment has not been established yet. ${ }^{1}$ Bilateral oopherectomy is supported by some authors, but more studies are required to confirm it. ${ }^{1,22}$ In our case, colectomy and metastectomy took place and it seems that metastectomy prolongs the mediate survival time and reduces the possibilities of the relapse, but the bilateral SO seems to have a good impact on the overall survival. ${ }^{22}$

As already mentioned, it was very difficult to clarify the primary site of the neoplasm. The immunohistochemical findings, usually, play a key role to the differential diagnosis, so the ovarian tumor, in our case, is immunoreactive for CK20 and negative for CK7 which means that is much more likely to be of colorectal in origin. ${ }^{3}$

Despite the fact that chemotherapy has been initially questioned as an adjuvant treatment, it seems nowadays, to have survival benefits. ${ }^{1,14}$ Capecitabine/ irinotecan combination regimens seem to provide advantages over the use of other chemotherapy treatments for metastatic colorectal cancer, so their mechanism and their effect is under consideration. ${ }^{23}$

Our patient has shown no complications since 30 months after the operation. The average survival time is between 3 and 10 months, indicating the extremely poor prognosis. ${ }^{2}$ So it is worth noticing some positive and negative prognostic factors in KT with colorectal origin. ${ }^{15}$ Two recent studies show that the primary site is the most important "prognostic factor" and, in fact, the prognosis is based on the origin. ${ }^{15,24}$ The synchronous diagnosis, the "ovarian-only" metastasis and the low pre-operative serum CA-125 level seem to be very good prognostic factors in our case of colorectal origin. ${ }^{1,3,15,17}$

\section{Conclusion}

Our case report is the proper paradigm of the necessity for more studies about the appropriate surgical treatment for KT. In our case, excision of both ovaries did not take place, but still the patient recovers well. Certain prognostic factors are associated with the survival of the patient and they should have more extensive practical use preoperatively and postoperatively.

\section{Acknowledgments}

None.

\section{Conflicts of interest}

The author declares that they do not have any conflicts of interest.

\section{References}

1. Al-Agha OM, Nicastri AD. An In-depth Look at Krukenberg Tumor: An Overview. Arch Pathol Lab Med. 2006;130(11):1725-30.

2. Das S, Sahu D, Wani M, et al. A curious discourse of Krukenberg tumor: a case report. J Gastrointest Oncol. 2014;5(6):E117-20.

3. Hatwal D, Joshi C, Chaudhari S, et al. Krukenberg tumor in a young woman: a rare presentation. Indian J Pathol Microbiol. 2014;57(1):124-6.

4. Young RH. From krukenberg to today: the ever present problems posed by metastatic tumors in the ovary: part I. Historical perspective, general principles, mucinous tumors including the krukenberg tumor. Adv Anat Pathol. 2006;13(5):205-27.

5. Tan KL, Tan WS, Lim JF, et al. Krukenberg tumors of colorectal origin: a dismal outcome--experience of a tertiary center. Int $J$ Colorectal Dis. 2010;25(2):233-8.

6. Man M, Cazacu M, Oniu T. Krukenberg tumors of gastric origin versus Krukenberg tumors of colorectal origin. Chirurgia (Bucur). 2007;102(4):407-10.

7. Ayhan A, Guney I, Saygan-Karamursel B, et al. Ovarian metastasis of primary biliary and gallbladder carcinomas. Eur J Gynaecol Oncol. $2001 ; 22(5): 377-8$.

8. Bilbao I, Vilallonga R, Allende E, et al.: Krukenberg's tumor as the first clinical manifestation of fibrolamellar hepatocarcinoma. Gastroenterol Hepatol. 2008;31(6):341-6.

9. Ebisui C, Ohkubo K, Akitake $\mathrm{H}$, et al. A case of ovarian metastasis from colon cancer successfully treated with multidisciplinary therapy. Gan To Kagaku Ryoho. 2010;37(12):2542-4.

10. Singhal SR, Nanda S, Chaudhry P, et al. Metastatic bilateral malignant ovarian tumors associated with pregnancy. Taiwan J Obstet Gynecol. 2009;48(2):167-8.

11. Sandmeier D, Lobrinus JA, Vial Y, et al. Bilateral Krukenberg tumor of the ovary during pregnancy. Eur J Gynaecol Oncol. 2000;21(1):58-60.

12. Stojnic J, Stefanovic A, Jeremic K, et al. Krukenberg tumor of gastric origin in pregnancy with dismal outcome. Eur J Gynaecol Oncol. 2011;32(3):356-8.

13. Papakonstantinou E, Liapis A, Kairi-Vassilatou E, et al. Virilizing ovarian Krukenberg tumor in a 27-year-old pregnant woman. A case report and literature review. Eur J Gynaecol Oncol. 2011;32(3):331-3. 
14. Wu F, Zhao X, Mi B, et al. Clinical characteristics and prognostic analysis of Krukenberg tumor. Mol Clin Oncol. 2015;3(6):1323-8.

15. Jeung YJ, Ok HJ, Kim WG, et al. Krukenberg tumors of gastric origin versus colorectal origin. Obstet Gynecol Sci. 2015;58(1):32-9.

16. Aktürk E, Dede M, Yenen MC, et al. Comparison of nine morphological scoring systems to detect ovarian malignancy. Eur J Gynaecol Oncol. 2015;36(3):304-8.

17. Sakakura C, Hagiwara A, Yamazaki J, et al. Management of postoperative follow-up and surgical treatment for Krukenberg tumor from colorectal cancers. Hepatogastroenterology. 2004;51(59):1350-3.

18. Gargano G, Catino A, Correale M, et al. Krukenberg tumor. A report of six cases. Eur J Gynaecol Oncol. 1992;13(5):431-5.

19. Hirschfield LS, Kahn LB, Winkler B, et al. Adenocarcinoid of the appendix presenting as bilateral Krukenberg's tumor of the ovaries. Immunohistochemical and ultrastructural studies and literature review. Arch Pathol Lab Med. 1985;109(10):930-3.
20. Miller RT, Sarikaya H, Jenison EL. Adenocarcinoid tumor of appendix presenting as unilateral Krukenberg tumor. J Surg Oncol. 1988;37(1):6571

21. Mandai M, Konishi I, Tsuruta Y, et al. Krukenberg tumor from an occult appendiceal adenocarcinoid: a case report and review of the literature. Eur J Obstet Gynecol Reprod Biol. 2001;97(1):90-5.

22. Erroi F, Scarpa M, Angriman I, et al. Ovarian metastasis from colorecta cancer: prognostic value of radical oophorectomy. J Surg Oncol. 2007;96(2):113-7.

23. Kerr DJ. Capecitabine/irinotecan combination regimens in colorectal cancer. Oncology (Williston Park). 2002;16(4 Suppl 3):27-9.

24. Guzel AB, Gulec UK, Paydas S, et al. Preoperative evaluation, clinical characteristics, and prognostic factors of nongenital metastatic ovarian tumors: review of 48 patients. Eur J Gynaecol Oncol. 2012;33(5):493-7. 